\title{
Stochastic Facilitation of Artificial Tactile Sensation in Primates
}

\author{
Leonel E. Medina, ${ }^{1,2}$ Mikhail A. Lebedev, ${ }^{2,3}$ Joseph E. O'Doherty, ${ }^{1,2}$ and Miguel A. L. Nicolelis ${ }^{1,2,3,4,5}$ \\ ${ }^{1}$ Department of Biomedical Engineering, ${ }^{2}$ Center for Neuroengineering, ${ }^{3}$ Department of Neurobiology, and ${ }^{4}$ Department of Psychology and Neuroscience, \\ Duke University, Durham, North Carolina 27710, and ${ }^{5}$ Edmond and Lily Safra International Institute of Neuroscience of Natal, 59066-060 Natal, Brazil
}

Artificial sensation via electrical or optical stimulation of brain sensory areas offers a promising treatment for sensory deficits. For a brain-machine- brain interface, such artificial sensation conveys feedback signals from a sensorized prosthetic limb. The ways neural tissue can be stimulated to evoke artificial sensation and the parameter space of such stimulation, however, remain largely unexplored. Here we investigated whether stochastic facilitation (SF) could enhance an artificial tactile sensation produced by intracortical microstimulation (ICMS). Two rhesus monkeys learned to use a virtual hand, which they moved with a joystick, to explore virtual objects on a computer screen. They sought an object associated with a particular artificial texture (AT) signaled by a periodic ICMS pattern delivered to the primary somatosensory cortex (S1) through a pair of implanted electrodes. During each behavioral trial, aperiodic ICMS (i.e., noise) of randomly chosen amplitude was delivered to S1 through another electrode pair implanted $1 \mathrm{~mm}$ away from the site of AT delivery. Whereas high-amplitude noise worsened AT detection, moderate noise clearly improved the detection of weak signals, significantly raising the proportion of correct trials. These findings suggest that SF could be used to enhance prosthetic sensation.

\section{Introduction}

Perhaps somewhat counterintuitively, combining informative signals with noise can enhance signal processing in biological systems (Moss et al., 2004). This phenomenon is referred to as stochastic facilitation (SF) or stochastic resonance (McDonnell and Ward, 2011). SF occurs when moderate levels of noise increase the number of neural threshold crossings, but also keep those crossings in synchrony with the signal (Moss et al., 2004). More intense noise masks the signal and worsens its detection. Examples of SF in human sensory processing include improved detection of visual stimuli (Kitajo et al., 2003), as well as mechanical stimuli applied to the fingers (Collins et al., 1996) and the foot sole (Wells et al., 2005). Similarly, SF has been reported for the cercal sensory system of crickets (Levin and Miller, 1996), CA1 neurons in hippocampal slices from rats (Stacey and Durand, 2001), and the spinal circuits of the cat (Martínez et al., 2007).

Received July 2, 2012; revised Aug. 10, 2012; accepted Aug. 24, 2012.

Author contributions: L.E.M., M.A.L., J.E.O., and M.A.L.N. designed research; L.E.M. and M.A.L. performed research; L.E.M., M.A.L., and M.A.L.N. analyzed data; L.E.M., M.A.L., and M.A.L.N. wrote the paper.

This work was supported by NIH Grants DP10D006798 and RC1HD063390 to M.A.L.N. The document is solely the responsibility of the authors and does not necessarily represent the views of the Office of the Director or NIH. We are grateful to Gary Lehew and Jim Meloy for engineering the experimental setup and the multielectrode arrays, Dragan Dimitrov and Laura Oliveira for conducting neural surgery, Solaiman Shokur for avatar design, Zheng Li for assistance in the decoding algorithm, Tamara Phillips for experimental support, and Susan Halkiotis for administrative assistance and editing the manuscript.

The authors declare no financial conflicts of interest.

Correspondence should be addressed to Miguel A. L. Nicolelis, Department of Neurobiology, Box 3209, Room 327E, Bryan Research Building Duke University Medical Center, 101 Research Drive, Durham, NC 27100. E-mail: nicoleli@neuro.duke.edu.

J.E. O'Doherty's present address: Department of Physiology and Center for Integrative Neuroscience, University of California, San Francisco, San Francisco, CA.

DOI:10.1523/JNEUROSCI.3115-12.2012

Copyright $\odot 2012$ the authors $\quad 0270-6474 / 12 / 3214271-05 \$ 15.00 / 0$
However, there is an ongoing debate as to whether SF serves any positive function in the neural circuitry (McDonnell and Abbott, 2009; McDonnell and Ward, 2011). Additionally, it is unclear whether SF could be used in assistive devices for people with sensory disabilities, for example, systems for sensory substitution (Bach-y-Rita and Kercel, 2003) and neural prosthetics with artificial sensation (Romo et al., 1998; O’Doherty et al., 2011).

Recently we described a sensorized neural prosthetic, called BMBI (brain-machine-brain interface), where neuronal activity recorded in the primary motor cortex (M1) was used to control the movements of a virtual arm while artificial tactile feedback from such an actuator was generated by intracortical microstimulation (ICMS) patterns delivered directly to somatosensory cortex (S1) (O’Doherty et al., 2009, 2011, 2012). This paradigm opens the possibility of aiding future human users of neuroprosthetic limbs with sensory signals arising from a variety of limb sensors. However, to develop sensorized neural prostheses further, detailed exploration is needed into ICMS as the means to reproduce the exteroception and proprioception normally provided by somatic sensation. Of particular interest is an inquiry into the processing of sensations evoked by ICMS when they are mixed with noisy inputs. For natural sensation, this problem is routinely solved by biological organisms in real-life environments (Moss et al., 2004). For artificial sensation, however, the research is only starting (O'Doherty et al., 2012). In the present study, we hypothesized that SF could enhance detection of weak ICMS patterns under an active exploration paradigm (O'Doherty et al., 2011; 2012). Accordingly, we delivered mixtures of stimulus and noise to $\mathrm{S} 1$ in different proportions and tested how their different combinations affected the subject's active exploration of artificial textures (ATs) 
A

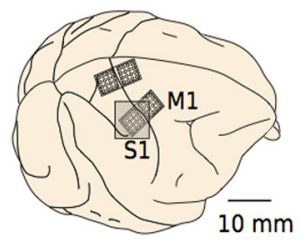

B

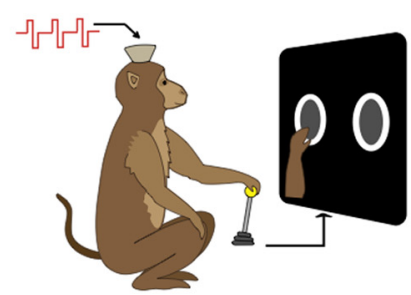

C 1 . Hold at center -0.5-1 s—

2. Go

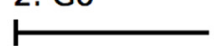

\section{Explore}

$10 \mathrm{~s}$

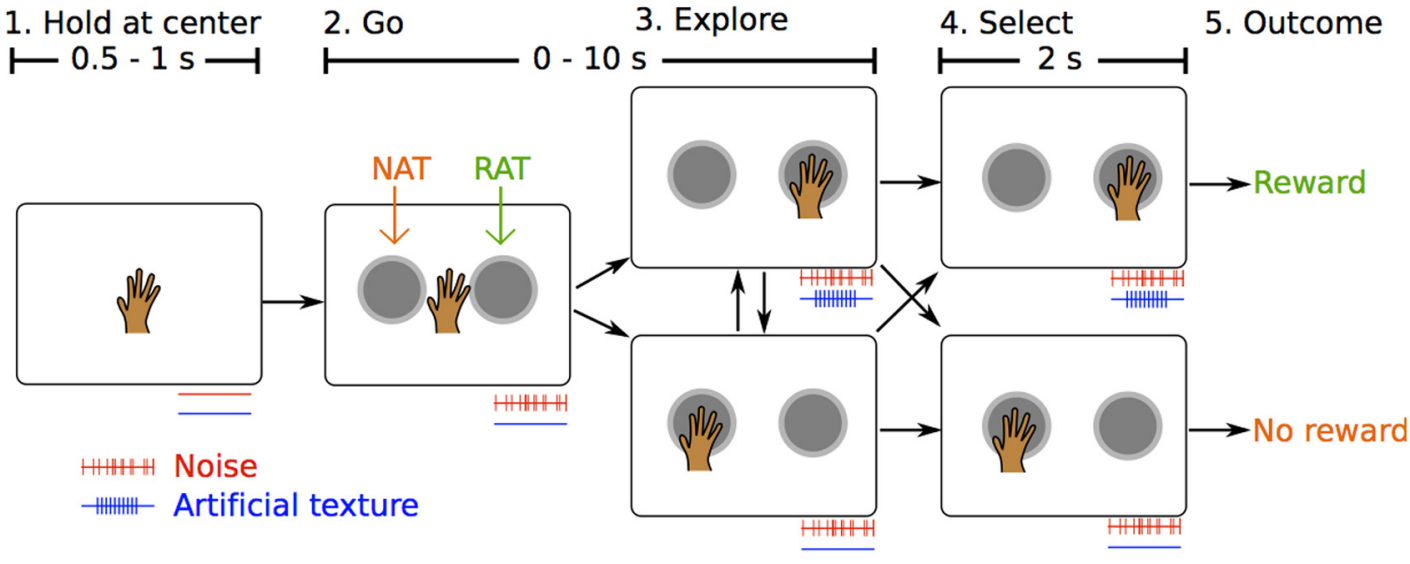

4. Select $2 \mathrm{~s}$

5. Outcome

Figure 1. Experimental setup and task schematics. $\boldsymbol{A}$, Arrays of microelectrodes were implanted in $\mathrm{S} 1$ and $\mathrm{M} 1$. Stimulating electrodes for monkey M (inset) are highlighted in red (AT) and yellow (noise). $\boldsymbol{B}$, Monkeys manipulated a joystick to move a virtual arm and reach toward objects on a computer screen. $\boldsymbol{C}$, Experimental task sequence. In the illustrated case, the selection of the right-hand object resulted in reward. The activity of both AT and noise ICMS channels are shown below each box.

A
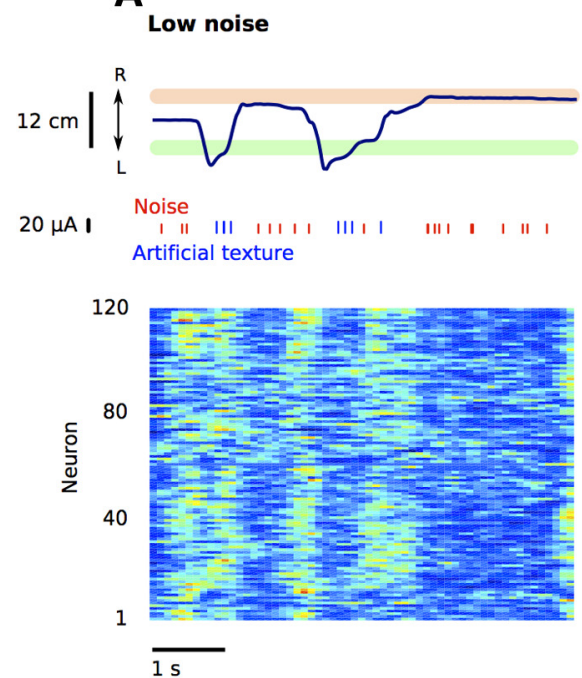

B

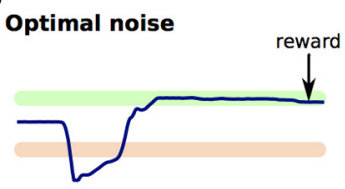

|| || | |||||||||||||||||||||||||

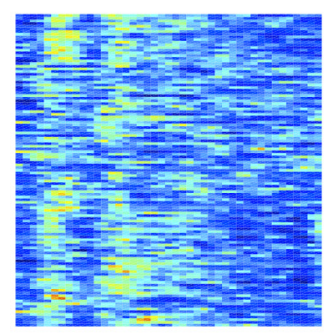

C High noise
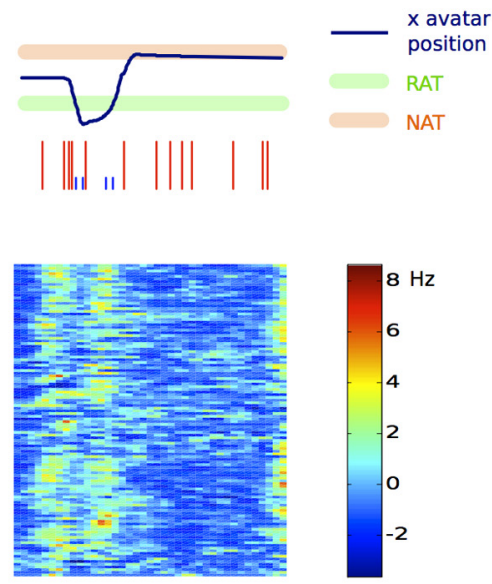

Figure 2. Detection of signal in noise and M1 modulations. Example traces of horizontal movement of the virtual hand for three trials with different noise levels: low $(\boldsymbol{A})$, optimal $(\boldsymbol{B})$, and high (C). The colored horizontal bars represent the position of the targets (green, RAT; brown, NAT). In the trials shown in $\boldsymbol{A}$ and $\boldsymbol{C}$, the monkey incorrectly selected the NAT, whereas in $\boldsymbol{B}$ it correctly picked the RAT and received a reward (indicated by a black arrow). The pulses delivered through both stimulating channels are shown. The RAT is subthreshold. The corresponding neuronal ensemble activity (monkey M, 120 units from M1) is shown in the bottom plots. Note that neuronal modulations reflect the speed and direction of arm movements. The color scale shows normalized firing rate (in $\mathrm{Hz}$ ).

\section{Materials and Methods}

Two rhesus monkeys, $\mathrm{M}$ (male) and $\mathrm{N}$ (female), were chronically implanted with cortical stainless steel microelectrode arrays in M1 and S1 in both hemispheres (Fig. 1A) (Nicolelis et al., 2003). Neuronal activity was sampled in the arm and hand areas of M1 and S1 in the hemisphere contralateral to the working arm. We used four electrodes of the S1 implants for ICMS delivery. Stimulation was applied to the right hemisphere arm area of S1 in monkey $\mathrm{M}$ and the right hemisphere leg area of $\mathrm{S} 1$ in monkey $\mathrm{N}$ using a custom-built, four-channel, current-controlled stimulator (Hanson et al., 2012). The monkeys were trained to move an image of the forearm and the hand (animated using MOTIONBUILDER; Autodesk) on a computer screen with a joystick (Fig. 1B). Using this virtual hand, they explored virtual objects presented on the screen, which were visually identical, yet differed in their ATs. Each trial started with the monkeys placing the virtual hand over a circular target that appeared at the center of the screen. At this time, ICMS noise of randomly chosen intensity started and continued until the end of the trial. Following the initial central hold, two visual objects appeared on the left and on the right of the screen, equidistant from the center. Then, the monkeys freely explored each of these objects to find the one associated with a periodic 
A
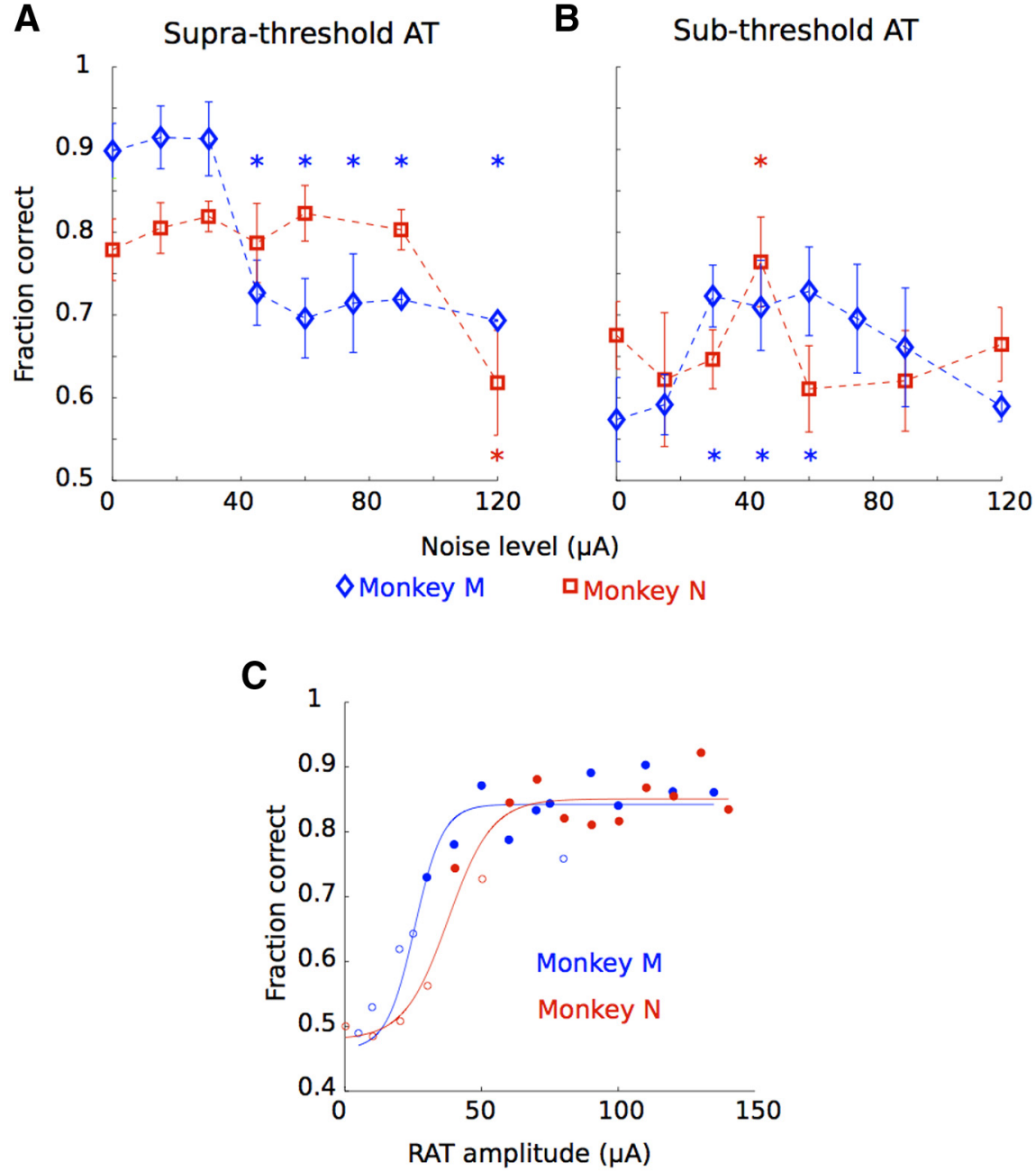

Figure 3. Stochastic facilitation via ICMS. $\boldsymbol{A}, \boldsymbol{B}$, Fraction of trials correct per session in terms of the noise amplitude level for monkey M (blue diamonds) and N (red squares) for suprathreshold $(\boldsymbol{A})$ and subthreshold $(\boldsymbol{B})$ RAT. ${ }^{*}$ Significantly different from average performance ( $p<0.05$ in all cases, randomization test). $\boldsymbol{C}$, Psychometric curves (in terms of ICMS amplitude) for monkeys $M$ (blue) and N (red). Lines, Best sigmoidal fits; open circles, performance at chance level ( $p>0.05$, Wilcoxon rank sum test).

that point. We then measured the psychometric threshold for RAT detection in the absence of noise for each monkey. Current amplitudes in the range 5-150 $\mu \mathrm{A}$ were tested. The detection threshold (Gescheider, 1997) was evaluated as the current level at which the proportion of correct responses was 0.75 . The psychometric curve (see Fig. 3C) for monkey M was slightly shifted to the left compared with that of monkey $\mathrm{N}$ and therefore its threshold was lower (31 vs $45 \mu \mathrm{A}$ for monkeys $\mathrm{M}$ and $\mathrm{N}$, respectively).

In subsequent sessions, ICMS noise was presented throughout each trial. Noise amplitude for each trial was randomly selected in multiples of $15 \mu \mathrm{A}$, up to $120 \mu \mathrm{A}$. Data with noise were collected for eight and nine daily sessions for monkeys $\mathrm{M}$ and $\mathrm{N}$, respectively (monkey $\mathrm{M}$ : $370 \pm 60$; monkey $\mathrm{N}: 418 \pm 87$ trials per session). For these sessions, the RAT amplitude was either suprathreshold $(50 \mu \mathrm{A})$ or subthreshold $(20 \mu \mathrm{A})$. Representative trials for a subthreshold stimulus combined with different noise intensities are shown in Figure 2. In these examples, the monkey correctly detected the subthreshold RAT $(20 \mu \mathrm{A})$ in the presence of an optimal noise $(30 \mu \mathrm{A})$ (Fig. $2 B)$, while failing to do so when the noise amplitude was too low (15 $\mu$ A; Fig. $2 A)$ or too high (90 $\mu$ A; Fig. 2C). Figure 2 also depicts the neuronal ensemble activity recorded in M1. The firing rates of M1 neurons were clearly modulated in association with the arm movements and reflected movement speed and direction.

Figure $3 A$ shows the fraction of correct detection (averaged by session) as a function of noise amplitude for suprathresh-

ICMS pattern, which we call the rewarded artificial texture (RAT). The other object lacked such ICMS feedback [null artificial texture (NAT)]. The RAT consisted of pulse pairs (with $10 \mathrm{~ms}$ interpulse interval for monkey $\mathrm{M}$ and $5 \mathrm{~ms}$ for monkey $\mathrm{N}$ ) delivered at a frequency of $10 \mathrm{~Hz}$. The monkey selected one of the objects by holding the virtual hand over it for $2 \mathrm{~s}$, and a juice reward was given if they correctly selected the RAT object (Fig. 1C). Each ICMS pulse was a symmetric, biphasic, chargebalanced current waveform delivered through a pair of electrodes implanted $3 \mathrm{~mm}$ from each other (i.e., bipolar stimulation). For noise delivery, a different electrode pair was used, implanted $1 \mathrm{~mm}$ away from the first pair of electrodes (Fig. 1A). The interpulse interval of such an aperiodic signal was drawn from a gamma distribution with the coefficient of variation (ratio of the standard deviation to the mean) equal to 1 (O’Doherty et al., 2012).

All animal procedures were performed in accordance with the $\mathrm{Na}$ tional Research Council's Guide for the Care and Use of Laboratory Animals and were approved by the Duke University Institutional Animal Care and Use Committee.

\section{Results}

Monkeys $\mathrm{M}$ and $\mathrm{N}$ were previously overtrained in a similar active exploration task (O'Doherty et al., 2011), so it took them just one and three training sessions, respectively, to achieve $~ 90 \%$ accuracy in the two-target task of this study. No noise was presented at old RATs $(50 \mu \mathrm{A})$. Note that in the absence of noise, monkey $\mathrm{M}$ performed better $(89.8 \%$ correct) than monkey $\mathrm{N}(77.9 \%$ correct), which is consistent with a more rapid saturation of the psychometric curve for monkey M (Fig. 3C). For monkey M, the performance decayed from this high level after noise amplitude exceeded $30 \mu \mathrm{A}$ [significantly lower success rate compared with the average for each noise level $>30 \mu \mathrm{A}, p<0.05$, randomization test (Edgington and Onghena, 2007)]. It is possible that we only observed decay simply because the performance was close to saturation from the start and could not improve in principle. However, for monkey $\mathrm{N}$, whose performance level had room for improvement, we observed a similar effect: the performance was resistant to weak noise and started to deteriorate when noise amplitude exceeded $120 \mu \mathrm{A}$ ( $p<0.001$, randomization test).

Changes in the monkey's detection performance with noise addition were very different when subthreshold RATs $(20 \mu \mathrm{A})$ were used. This dependency was characterized by a nonmonotonic curve with peaks at noise amplitude of 60 and $45 \mu \mathrm{A}$ for monkeys $\mathrm{M}$ and $\mathrm{N}$, respectively. Thus, for monkey $\mathrm{M}$, elevated performance of $\sim 0.7$ was observed for three noise amplitudes: 30,45 and $60 \mu \mathrm{A}(p<0.05$ for all points, randomization test). For monkey $\mathrm{N}$, the curve had a single peak of 0.76 at $45 \mu \mathrm{A}(p<$ 
0.01 , randomization test). Therefore, in both monkeys, the addition of a moderate level of ICMS noise to a weak AT stimulus improved the animal's detection performance. Furthermore, neither the presence of ATs nor the addition of noise substantially affected movement-related modulations observed in M1 (Fig. 2), nor did it degrade the performance of a decoding algorithm that extracted the position of the virtual arm from the population activity of M1 neurons [unscented Kalman filter (Li et al., 2009); monkey M: 125 units, monkey N: 65 units; Fig. 4].

\section{Discussion}

The present findings demonstrate for the first time SF effects for an artificial tactile sensation. The ability of monkeys to detect weak ICMS signals was clearly improved by the addition of moderate levels of ICMS noise. This result is consistent with previous model and experimental demonstrations of SF in physiological systems (Moss et al., 2004). Interest in SF for neural systems has dramatically increased over the last 20 years, driven by theories claiming that noise may play an important role in normal neural processing (McDonnell and Abbott, 2009; McDonnell and Ward, 2011). Since the presence of moderate amounts of internal noise may be normal and even advantageous for neural systems, several publications proposed implementing noise and SF in artificial systems designed to restore normal functions to people with disabilities, for example in cochlear implants (Morse et al., 2007), assistive devices for postural balance (Harry et al., 2005), and hand sensorimotor tasks (Kurita et al., 2011). Here we demonstrated the practicality of this idea for prosthetic sensation evoked by ICMS. By adding background noise, we effectively gained control over the detection threshold for an artificial tactile sensation. It has yet to be determined how SF would work in the case of prosthetic sensations evoked by spatiotemporal input patterns delivered through multielectrode implants (Fitzsimmons et al., 2007). For such multichannel systems with stimulation sites distributed over the cortical surface, the superposition of spatiotemporal noise patterns could enhance signal detection in both the temporal and spatial dimensions. Furthermore, SF patterns distributed across corticothalamic loops could be obtained in sensorized neural prosthetics that use thalamic stimulation as an additional channel for artificial somatic sensation (Heming et al., 2010). Although in our present study we did not manipulate the statistics of the noise channel, it would be of great interest in the future to examine a variety of noise-frequency characteristics. If noise contains particular frequencies, SF may accordingly filter frequency components of the input signal. Importantly for practical neuroprosthetic systems, the potential risks of neural damage associated with ICMS may be lower for a SF-based neural prosthetic because of the lower currents injected into the neural tissue (McCreery et al., 2010). In conclusion, we suggest that the incorporation of SF effects in sensorized neural prosthetics may enhance a variety of functional capabilities.

\section{References}

Bach-y-Rita P, Kercel SW (2003) Sensory substitution and the humanmachine interface. Trends Cogn Sci 7:541-546. CrossRef Medline

Collins JJ, Imhoff TT, Grigg P (1996) Noise-enhanced tactile sensation. Nature 383:770. CrossRef Medline

Edgington ES, Onghena P (2007) Randomization tests, 4th ed. Boca Raton, FL: CRC.

Fitzsimmons NA, Drake W, Hanson TL, Lebedev MA, Nicolelis MA (2007) Primate reaching cued by multichannel spatiotemporal cortical microstimulation. J Neurosci 27:5593-5602. CrossRef Medline

Gescheider GA (1997) Psychophysics: the fundamentals, 3rd ed. Mahwah, NJ: Lawrence Erlbaum Associates.

Hanson TL, Ómarsson B, O’Doherty JE, Peikon ID, Lebedev MA, Nicolelis MA (2012) High-side digitally current controlled biphasic bipolar microstimulator. IEEE Trans Neural Syst Rehabil Eng 20:331-340. CrossRef Medline

Harry JD, Niemi JB, Priplata AA, Collins JJ (2005) Balancing act [noise based sensory enhancement technology]. IEEE Spectr 42:36-41. CrossRef

Heming E, Sanden A, Kiss ZH (2010) Designing a somatosensory neural 
prosthesis: percepts evoked by different patterns of thalamic stimulation. J Neural Eng 7:064001. CrossRef Medline

Kitajo K, Nozaki D, Ward LM, Yamamoto Y (2003) Behavioral stochastic resonance within the human brain. Phys Rev Lett 90:218103. CrossRef Medline

Kurita Y, Shinohara M, Ueda J (2011) Wearable sensorimotor enhancer for a fingertip based on stochastic resonance. Paper presented at 2011 IEEE International Conference on Robotics and Automation, Shanghai, China, May 9-13. CrossRef

Levin JE, Miller JP (1996) Broadband neural encoding in the cricket cercal sensory system enhanced by stochastic resonance. Nature 380:165-168. CrossRef Medline

Li Z, O’Doherty JE, Hanson TL, Lebedev MA, Henriquez CS, Nicolelis MA (2009) Unscented Kalman filter for brain-machine interfaces. PLoS One 4:e6243. CrossRef Medline

Martínez L, Pérez T, Mirasso CR, Manjarrez E (2007) Stochastic resonance in the motor system: effects of noise on the monosynaptic reflex pathway of the cat spinal cord. J Neurophysiol 97:4007-4016. CrossRef Medline

McCreery D, Pikov V, Troyk PR (2010) Neuronal loss due to prolonged controlled-current stimulation with chronically implanted microelectrodes in the cat cerebral cortex. J Neural Eng 7:036005. CrossRef Medline

McDonnell MD, Abbott D (2009) What is stochastic resonance? Definitions, misconceptions, debates, and its relevance to biology. PLoS Comput Biol 5:e1000348. CrossRef Medline

McDonnell MD, Ward LM (2011) The benefits of noise in neural systems: bridging theory and experiment. Nat Rev Neurosci 12:415-426. CrossRef Medline

Morse RP, Morse PF, Nunn TB, Archer KA, Boyle P (2007) The effect of
Gaussian noise on the threshold, dynamic range, and loudness of analogue cochlear implant stimuli. J Assoc Res Otolaryngol 8:42-53. CrossRef Medline

Moss F, Ward LM, Sannita WG (2004) Stochastic resonance and sensory information processing: a tutorial and review of application. Clin Neurophysiol 115:267-281. CrossRef Medline

Nicolelis MA, Dimitrov D, Carmena JM, Crist R, Lehew G, Kralik JD, Wise SP (2003) Chronic, multisite, multielectrode recordings in macaque monkeys. Proc Natl Acad Sci U S A 100:11041-11046. CrossRef Medline

O’Doherty JE, Lebedev MA, Hanson TL, Fitzsimmons NA, Nicolelis MA (2009) A brain-machine interface instructed by direct intracortical microstimulation. Front Integr Neurosci 3:20. Medline

O’Doherty JE, Lebedev MA, Ifft PJ, Zhuang KZ, Shokur S, Bleuler H, Nicolelis MA (2011) Active tactile exploration using a brain-machine-brain interface. Nature 479:228-231. CrossRef Medline

O'Doherty JE, Lebedev MA, Li Z, Nicolelis MA (2012) Virtual active touch using randomly patterned intracortical microstimulation. IEEE Trans Neural Syst Rehabil Eng 20:85-93. CrossRef Medline

Romo R, Hernández A, Zainos A, Salinas E (1998) Somatosensory discrimination based on cortical microstimulation. Nature 392:387-390. CrossRef Medline

Stacey WC, Durand DM (2001) Synaptic noise improves detection of subthreshold signals in hippocampal CA1 neurons. J Neurophysiol 86:1104-1112. Medline

Wells C, Ward LM, Chua R, Timothy Inglis J (2005) Touch noise increases vibrotactile sensitivity in old and young. Psychol Sci 16:313-320. CrossRef Medline 